\title{
Impact of Confrontations by Therapists on Impairment and Utilization of the Therapeutic Alliance
}

Laura Moeseneder $^{\mathrm{a} *}$, Eugénia Ribeiro ${ }^{\mathrm{b}}$, John Christopher Muran ${ }^{\mathrm{c}}$ and Franz Caspar $^{\mathrm{a}}$

${ }^{a}$ Department of Clinical Psychology and Psychotherapy, Institute of Psychology, University of Berne, Berne, Switzerland

${ }^{b}$ Psychotherapy and Psychopathology Research Unit, Centre of Research in Psychology School of Applied Psychology, University of Minho, Braga, Portugal

${ }^{c}$ Gordon F. Derner Institute of Advanced Psychological Studies, Adelphi University, New York, USA; Brief Psychotherapy Research Program, Mount Sinai Beth Israel, New York, USA

*Laura Moeseneder, Institut für Psychologie, Fabrikstrasse 8

3012 Bern, Switzerland, laura.moeseneder@psy.unibe.ch, 0041316315424

Eugénia Ribeiro, Escola de Psicologia, Universidade do Minho, Campus de Gualtar, Braga, Portugal, eugenia@psi.uminho.pt, 351253604612

John Christopher Muran, Beth Israel Medical Center, Department of Psychiatry \& Behavioral Sciences, First Avenue at 16th Street, New York, New York 10003, USA, jcmuran@adelphi.edu, 5168774803

Franz Caspar, Institut für Psychologie, Fabrikstrasse 8, 3012 Bern, Switzerland, caspar@psy.unibe.ch, 0041316314731

This is an Accepted Manuscript of an article published by Taylor \& Francis in Psychotherapy Research on 26/07/201, available online: https://www.tandfonline.com/doi/full/10.1080/10503307.2018.1502897. 


\section{Impact of Confrontations by Therapists on Impairment and Utilization of the Therapeutic Alliance}

Objective: Striking the balance between creating challenge through confrontation (drawing attention to discrepancies) to encourage change and offering support through the therapeutic relationship to ensure safety for patients represents a central issue for psychotherapists. The aim of the present study was to assess immediate effects of confrontations by therapists on the therapeutic alliance. Method: We rated video recordings of 77 therapies to measure incidences of alliance ruptures/resolution attempts as well as confrontations by therapists. Change in the therapeutic alliance and therapy outcome were measured through questionnaires filled out by patients.

Results: Confrontations were significantly associated with ruptures/resolution attempts on the micro level. Changes in the therapeutic alliance moderated the association between confrontations and alliance ruptures on the macro level: The bigger the fraction of a session containing confrontations, the larger the fraction containing ruptures, given a prior positive change in the therapeutic alliance. Therapists' use of confrontation during a resolution attempt was associated with significantly better therapy outcomes than no use of confrontation during or no resolution attempt. Conclusions: Confrontations by therapists may temporarily impair the therapeutic alliance, but might also lead to better therapy outcomes when used to make an alliance rupture explicit as part of a resolution attempt.

\section{Disclosure Statement}

The authors report no conflicts of interest. 


\section{Introduction}

Striking the balance between creating challenge to encourage change and offering support to ensure a sense of safety in patients represents a central issue for psychotherapists. For example, therapists may find themselves in a dilemma between situating themselves within clients' values versus confronting clients with regard to problematic values (Williams \& Levitt, 2007). Therapists also frequently suspect that their own pushy or cautious interventions may have led to impasses in therapies (Hill, Nutt-Williams, Heaton, Thompson, \& Rhodes, 1996). Adopting a broad view, Caspar (2007) and Sachse (2003) proposed providing support through the safety of the therapeutic relationship, while challenging patients through interventions such as confrontation. These prescriptive models of balance mainly suggest to first provide security in the therapeutic relationship and to subsequently confront patients. Therapists also rely on such a model, when planning a confrontation. Thus, they try to secure the therapeutic relationship before a confrontation, so they might lower the probability of a momentary impairment in the therapeutic relationship afterwards (Moeseneder et al., 2017, Figure 1). Ribeiro, Ribeiro, Gonçalves, Horvath, and Stiles (2013) conceptualized the balance between challenge and support on a more microscopic level in moment-to-moment interactions. In their model, clients' experience of safety is induced by responsive and empathic therapists' interventions, independently of whether they support or challenge the clients’ perspectives or framework.

[Figure 1 near here]

Consequently, no matter the scale of the processes within models of balance, confrontation cannot be looked at separately as it seems to be intimately linked with the therapeutic relationship. The literature on this interplay is ambiguous: Boardman, Catley, Grobe, Little, and Ahluwalia (2006) found that confrontation had a negative influence on the therapeutic relationship, more specifically on the therapeutic alliance. Similarly, therapists in 
the study of Coutinho, Ribeiro, Hill, and Safran (2011) suspected untimely/inappropriate challenging interventions as a cause for impaired therapeutic alliance. On the other hand, Moyers, Miller, and Hendrickson (2005) have concluded that confrontations were positively related to client involvement, which may correlate with a good therapeutic relationship. Consequently, no clear and simple heuristic can be extrapolated from these studies to guide therapists in their struggle to balance support and challenge through confrontation.

\section{Defining Confrontations by Therapists and Changes in the Therapeutic Relationship}

Apart from inconsistent results, the above mentioned studies lacked methods to observe the close intertwining between confrontation and therapeutic relationship: Challenging interventions (such as confrontations) were operationalized through patients' and therapists' retrospective accounts (Coutinho et al., 2011) or the therapeutic relationship was measured indirectly (Moyers et al., 2005). Additionally, the definition of confrontation by therapists varied in all studies. Lastly, no studies measured both moment-to-moment changes in the therapeutic alliance and confrontations within sessions. As a possibility to measure the former, Eubanks, Muran, and Safran (2015) provide the Rupture Resolution Rating System (3RS) as a manual to capture momentary processes relating to the therapeutic alliance. The therapeutic alliance represents an important component of the therapeutic relationship, as described by Horvath (2000) and is defined by agreement on tasks as well as treatment goals and offers a personal bond between therapist and patient (Bordin, 1979). The 3RS allows the measurement of deteriorations in the alliance, known as alliance ruptures (Safran, Crocker, McMain, \& Murray, 1990) as well as therapist efforts to repair these ruptures, known as resolution attempts. On the other hand, a rating system focusing only on confrontation by therapists has not yet been established. This may be due to the heterogeneity of the definition of confrontation in the literature. 
To define confrontation for our study, we integrated two core topics in existing definitions. Firstly, we included the criterion of a focus on discrepancies by therapists (for examples see Hill, 1978 or Polcin, 2006) into the definition of the content of a confrontation. These discrepancies may lie within the patient (for instance, wish to enjoy social activities vs. fear of rejection in social settings), between patient and therapist (for example, therapist sees avoidance of social events as problematic vs. patient sees no need to stop avoidance) or between patient and patient's environment (for instance, patient wishes to go to plays with friends vs. friends are only interested in watching sports). Secondly, we included an expansion of patients' awareness as a frequently mentioned desirable consequence of a confrontation (e.g. Grawe, 2004; Meystre, Pascual-Leone, de Roten, Despland, \& Kramer, 2015 or Strong \& Zeman, 2010). Therefore, in this study, we define a confronting intervention as focusing on discrepancies that the patient may or may not be aware of. This includes the possibility of an already existing awareness of the patient that is supposed to be augmented or made more explicit through a confrontation. ${ }^{1}$

\section{Risks related to Confrontation}

Based on the above, therapists may induce insight but also potentially put strain on the therapeutic relationship when confronting patients. The concept of the therapeutic zone of proximal development (TZPD, Leiman \& Stiles, 2001; Ribeiro et al., 2013) provides concrete assumptions to explain such processes. Based on the TZPD, the therapist's job lies in working between a patient's actual developmental level that is characterized by limited capacity to cope with a specific problem and a patient's potential developmental level that

\footnotetext{
${ }^{1}$ To prevent confusion with confrontational alliance ruptures which are defined as a patient's direct expression of negative sentiments (e.g. in the 3RS), they will be clearly marked as such in this article, while the term confrontation refers to the therapist's intervention defined above.
} 
represents the capacity that can be reached in collaboration with the therapist. Therapists need to work within their clients' therapeutic zone of proximal development or the space between the two above-mentioned developmental levels. A successful confrontation within the TZPD would lead a patient to experience safety or tolerable risk. This encourages assimilative processes such as insight. An unsuccessful confrontation may be beyond the patient's potential level, thus exceeding the patient's TZPD and leading to experiences of ambivalence or even intolerable risk (translating into anxiety). The patient then invalidates the therapist's intervention, which might present itself as an alliance rupture, and be perceived as a hostile reaction. Therefore, it is critical for therapists to know beforehand whether a confrontation might push beyond a patient's potential developmental level. By anticipating the patient's reaction, the therapist can time or shape the intervention accordingly or even plan resolution attempts to repair potential alliance ruptures.

However, empirical results on this issue are lacking, because confrontation as an intervention has not been investigated as extensively as the therapeutic relationship that might be regarded as a common factor (e.g. Lambert \& Barley, 2001; Norcross, 2002). Moreover, the number of studies looking at the interplay between the therapeutic relationship and confrontation (Boardman et al., 2006; Coutinho et al., 2011; Moyers et al., 2005) is small and these studies lack methods to closely assess immediate risks of confrontation for the therapeutic relationship or more specifically, for the therapeutic alliance. To bridge this gap in knowledge, this study was designed to assess immediate effects of confrontations by therapists on the therapeutic alliance.

\section{Current Study}

We tested specific assumptions of therapists interviewed in a qualitative interview study conducted earlier (Moeseneder et al., 2017), because general models of balance differ in their prediction of alliance ruptures with confrontations, and results of past studies were 
inconsistent. Specifically, we first tested the two following hypotheses (see also Figure 1) by measuring confrontations and momentary noticeable changes in the therapeutic alliance through observer-based ratings of video recorded therapy sessions: 1 . Confrontations are positively associated with alliance ruptures when examining smaller sequences within therapy sessions (Micro level). 2. The positive association between confrontations and alliance ruptures is moderated by the previous quality of the therapeutic alliance when examining entire therapies (Macro level). This association between confrontations and ruptures disappears when there has been prior positive change in the therapeutic alliance.

As part of our analysis to test the first hypothesis, we found a parallel use of resolution attempts and confrontations by therapists. Furthermore, a meta-analysis (Safran, Muran, Samstag, \& Stevens, 2001) suggested a positive effect of rupture-repair episodes (alliance ruptures and corresponding resolution attempts) on therapeutic outcome. Therapists also assume that confrontation may induce insight and strengthen the therapeutic relationship either directly or - indirectly through the repair of an alliance rupture (Moeseneder et al., 2017) and therefore positively influence therapy outcome. Due to our first results and the mentioned literature, a post-hoc created hypothesis was additionally tested: 3 . Use of confrontation during resolution attempts leads to a better therapy outcome than using no confrontation during resolution attempts or using no resolution attempts at all when examining whole therapies (Macro level).

\section{Method}

Video recordings of 77 therapies held in German or Swiss German at the Psychotherapy Outpatient Clinic of the Institute of Psychology at the University of Bern in Switzerland were analyzed. These therapies were conducted between 2007 and 2017 (5 were still ongoing in January 2018) and were chosen to represent the whole population of the outpatient clinic based on patients' age, gender and diagnoses. The mean number of sessions per therapy was 
$40.15(S D=30.72$, range $=14-194$, median $=28.5)$. Video records from two different sessions were analyzed for each therapy. The videos were chosen from the middle third of therapy, because literature showed a higher probability for confrontations to occur around this time and authors recommend starting to confront patients at that time point of therapy (for examples see Claiborn, Goodyear, \& Horner, 2002 or Engle \& Arkowitz, 2008).

\section{Patients}

The majority (63\%) of the 77 participants were female, with a mean age of 39.89 years $(S D=$ 10.36, range 19 - 75). Patients were diagnosed with the German version of the SCID-IV² ( First, Spitzer, Gibbon, \& Williams, 1995a; First et al., 1995b;). The primary and secondary diagnoses of the sample included: affective disorders such as major depression or dysthymia (38 patients), anxiety disorders such as specific phobia (26 patients), adjustment disorders (14 patients) and no disorder (12 patients). Approximately a third (22 patients) were diagnosed with other disorders such as personality disorder (two histrionic, one borderline and one avoidant personality disorder diagnosis) or bulimia nervosa. Thirty patients received only one diagnosis. All participating patients gave their written consent to the inclusion of material pertaining to themselves. The material used in this study was fully anonymized.

\section{Therapists}

Six of the 24 therapists were experienced, 18 in training. The majority (15/24) were female, with a mean of 5.37 years of experience as practicing therapist $(S D=6.06$, range $=1-24)$. Therapists treated 3.21 patients on average (range $=2-7$ ). The common theoretical background of the therapists lays in integrative cognitive behavioral therapy (emphasis on cognitive-behavioral therapy with inclusion of empirically supported methods and concepts from other approaches, e.g. system therapy or emotion-focused therapy) complemented with

\footnotetext{
${ }^{2}$ Semi-structured interview guide relating to DSM-IV (American Psychiatric Association, 2000).
} 
"Bernese” concepts (Grawe, 2004; Caspar, 2007). In their daily work, the therapists used case conceptualizations that included Plan Analysis (Caspar, 2007) and Motive Oriented Therapeutic Relationship (MOTR; Caspar, 2007). MOTR is a prescriptive approach to create a solid therapeutic relationship and suggests behaving responsively to patients by furthering their most important motives and needs. These are deduced from Plan Analyses ${ }^{3}$. Plan Analysis focuses on the instrumentality between patients' verbal and nonverbal behavior and hierarchically depicts the motives and needs underlying the observed behavior.

\section{Measures}

\section{Incidence of Confrontations by Therapists}

The confrontation rating manual used in this study was largely based on the rating manual used in a doctoral thesis at the University of Bern (Figlioli, 2016). In its development, items were generated based on a literature review, discussed with the senior author of this manuscript and applied to specific therapy sequences on videotape in a testing phase. Items were further discussed with the respective therapists of the sessions and were slightly adapted. For the present study, duration and type of discrepancy of a confrontation were of interest. The raters were four master students. They first studied a representation of a patient's functioning based on Plan Analysis to notice confrontations regarding the patient's specific motives. Secondly, the raters noted moments of tension in the therapy session. Thirdly, during a second viewing they recorded ending as well as starting point of each confrontation and categorized the discrepancies pointed out by therapists during each confrontation (within patient, between patient and patient's environment or between patient and therapist). They transcribed all the relevant scenes. Ending and starting points of the

\footnotetext{
${ }^{3}$ The use of Plan Analyses and MOTR received empirical support in several studies (e.g. Grawe,
} Caspar, \& Ambühl, 1990; Kramer et al., 2014). 
confrontations were defined based on the time a therapist used to refer to the same discrepancy without changing the topic. As a consequence, confrontations could consist of a single statement by the therapist or various conversational turns. During training, the raters independently created ratings of the same sessions and discussed those ratings during meetings moderated by the first author of this study. These meetings took place over a time period of five months. To measure the reliability of the ratings, $18 \%$ (14 therapies) of the videos were coded twice; each rater was compared to all other raters and a mean was created based on comparisons between each rater pair. This procedure resulted in an intraclass correlation of .68 (fraction of a session occupied by confrontations) and a Cohen’s kappa of .67 (incidence of confrontation within a 5-minute-sequence). Both measures can be categorized as good (Cicchetti, 1994). The mean of the fraction of a session occupied by confrontation was calculated with the following formula and ranged from $0.40 \%$ to $19.56 \%$ $(M=3.82 \%, S D=3.32 \%):$

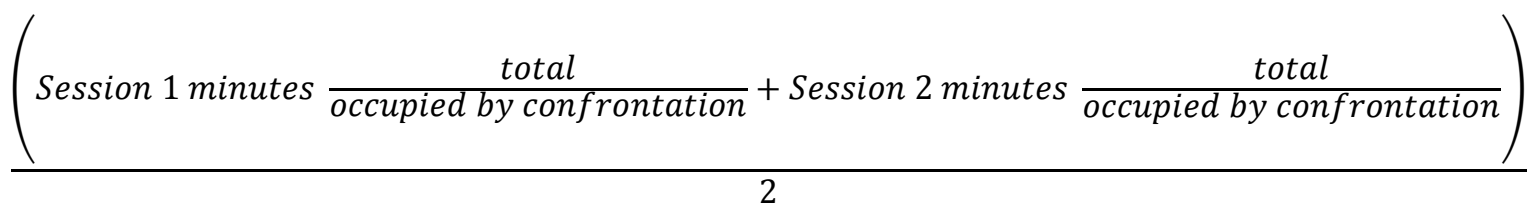

\section{Alliance Ruptures and Resolution Attempts}

Incidence of alliance ruptures as well as resolution attempts were rated by a different group of four master students. They used the Rupture Resolution Rating System (3RS) by Eubanks et al. (2015) and were trained in the same fashion as mentioned above over a period of three months. In the rating process, a rupture was categorized as either confrontational (when the patient moves against the therapist by expressing anger or dissatisfaction) or withdrawing (when the patient either moves away from the therapist or denies an aspect of his or her experience). Secondly, raters noted incidences of resolution attempts, which had to be in the context of a rupture. This usually meant that a rupture occurred prior to the resolution attempt in the same session. Alternatively, a therapist referred to a rupture from the first session in the 
second of the two investigated sessions. A mean of the fraction of a session occupied by ruptures (confrontational and withdrawing taken together) was calculated from the two rated sessions with the following formula:

$$
\frac{\left(\text { Session } 1 \text { sequences } \frac{\text { total }}{\text { occupied by ruptures }}+\text { Session } 2 \text { sequences } \frac{\text { total }}{\text { occupied by ruptures }}\right)}{2}
$$

The mean fraction of sessions occupied by ruptures ranged from $0.00 \%$ to $90.91 \%$ ( $M=$ $25.92 \%, S D=17.36 \%)$. Resolution attempts were taken together independently of rated success or specific resolution strategy. The reliability of the ratings based on the 3RS was measured and calculated in the same manner as mentioned above from 12 therapies (16\%). This procedure resulted in an intraclass correlation of .74 for alliance ruptures and .63 for resolution attempts, which can be considered good (Cicchetti, 1994). The Cohen's kappa was .46 (incidence of rupture and/or resolution attempt within a 5-minute-sequence) which can be categorized as fair (Cicchetti, 1994). This lower reliability in regard to the Cohen's kappa might have been influenced by the size of the rated units or the appearance of alliance ruptures in the examined Swiss/European population (Raters noted subtler presentation of alliance ruptures in the examined sample in comparison to the examples in the 3RS).

\section{Change in Global Alliance after Therapy Onset}

The Bern Post-Session Report for patients (BPSR, Flückiger, Regli, Zwahlen, Hostettler, \& Caspar, 2010) was administered after every therapy session. This has been routinely done in all archived sessions as part of ongoing research activity at the outpatient clinic. Internal consistency of BPSR scales ranges from .74 to .88 (Flückiger, Caspar, Holtforth, \& Willutzki, 2009). The questionnaire comprised 26 items (all originally in German), of which 7 items captured the patient's view on the global alliance (examples: “My therapist and I get along well.” or “The therapist understood and supported me in my most important needs.”) and were rated based on a 7-point Likert scale ranging from 0 (not at all) to 6 (completely 
right). The difference of these 7 items between the start of the therapies (sessions " 1 ”, “2” and “3”) and immediately before the two investigated sessions (sessions “-3”, “-2” and “-1”) was calculated to operationalize change in the global alliance after therapy onset as following:

$$
\left(\text { Alliance } \frac{(\text { Session }-3+-2+-1)}{3}\right)-\left(\text { Alliance } \frac{(\operatorname{Session} 1+2+3)}{3}\right)
$$

A positive score indicated a positive change, while a negative score indicated a negative change. We decided against a cut-off level to operationalize a sufficient global alliance, because therapists stated in a prior study (Moeseneder et al., 2017) that the therapeutic relationship before a confrontation needs to be secured. Therefore, the development of security in the global alliance was defined as a positive change after therapy onset. The change in global alliance ranged from -1.07 to 1.10 points $(M=0.07, S D=0.45)$.

\section{Therapy Outcome}

The outcome of therapy was operationalized by change in measures of success from the time around the two investigated sessions (intermediate measures) and post-therapy measures. Intermediate measures have been gathered in all archived therapies of the outpatient clinic repeatedly in intervals of approximately ten sessions as part of ongoing research and evaluation activity. Video recordings of the sessions directly before the intermediate measures could not be found or were not of satisfactory quality (e.g. missing audio) in 4 therapies. In these cases measurements happened directly after (in two therapies) or between (in the other two therapies) the investigated sessions. Ten therapies lacked all intermediate measures. All instruments were based on patient self-reports and encompassed the following five measures: Goal Attainment Scaling (GAS, Cardillo \& Smith, 1994), Self-Efficacy (SE, Jerusalem \& Schwarzer, 1999), Inconsistency (INC, Grosse Holtforth \& Grawe, 2003), Short version of the Symptom Check List-90 (SCL-90, Klaghofer \& Brähler, 2001) and Change Questionnaire in Life Domains (SQL, Itten \& Grawe, 2003). All five measures were included 
to ensure satisfactory statistical power, as fewer measures could not provide sufficient data due to the naturalistic setting of our study (e.g. a change in measures at the outpatient clinic around the middle of our observation period which lead to missing data regarding the SCL-90 in approximately a fourth of the sample). A standardized mean difference ${ }^{4}$ was calculated for every patient as following:

$$
\begin{aligned}
& \begin{array}{c}
\text { GAS } \frac{\text { post-intermediate }}{\text { pooled Std.Dev. }}+S E \frac{\text { post-intermediate }}{\text { pooled Std.Dev. }}+I N C \frac{\text { intermediate-post }}{\text { pooled Std.Dev. }}+ \\
\text { SCL-90 } \frac{\text { intermediate-post }}{\text { pooled Std.Dev. }}+S Q L \frac{\text { post-intermediate }}{\text { pooled Std.Dev. }}
\end{array} \\
& 5
\end{aligned}
$$

These effect sizes can be interpreted as small $(=0.2)$, medium $(=0.5)$ or large $(=0.8)$ based on Cohen (1992). Missing data on singular measures were ignored and the mean was calculated based on the remaining measures.

\section{Statistical Analyses}

Hypothesis 1 (Micro Level): Confrontations associated with Alliance Ruptures

To probe the association between confrontations by therapists and noticeable changes in the therapeutic alliance, a generalized linear mixed-effects model (GLMM) analysis was conducted. We predicted incidence of therapists' confrontations (taking together all three types of discrepancies described above) with noticeable changes in the therapeutic alliance (ruptures and/or resolutions attempts) per each five-minute sequence in the two sessions per therapy. The binary outcome (incidence of confrontation given or not) was predicted with a variable with four categories: nothing (no ruptures/no resolution attempts), rupture only, resolution attempt only and rupture-resolution episode (rupture and associated resolution

\footnotetext{
${ }^{4}$ Considering controversy around residualized change scores (e.g. Fitzmaurice, Laird, \& Ware, 2012 or Gollwitzer, Christ, \& Lemmer, 2014) we opted to use the standardized mean difference as it provides simpler clinical implications.
} 
attempt within same five-minute sequence). The analysis allowed to control for clustering by patients through matching various five-minute sequences on level 1 to the mutually corresponding patient on level 2. The software R (R Development Core Team, 2014) with the package Ime4 (glmer procedure, Bates, Mächler, Bolker, \& Walker, 2014) was used for the estimations.

\section{Hypothesis 2 (Macro Level): Moderation}

A linear mixed model was estimated with the lme4 Package in $\mathrm{R}$ (lmer procedure, Bates et al., 2014) to account for the clustering of patients (level 1 units) by therapists (level 2 units). In a random intercept model, the mean fraction of a session occupied by ruptures was predicted with three variables: 1. mean fraction of a session occupied by confrontations, 2 . change in the global alliance after therapy onset and, 3. an interaction term created with the two previously mentioned variables. Confrontations that focused on discrepancies between therapist and patient, which were used in the same sequence as a resolution attempt were excluded based on the results of the analysis regarding hypothesis 1. Data from two therapies (two different therapists) regarding the global alliance were missing, so they were deleted listwise for this analysis. Predictors were centered with the group mean (= one mean per therapist) to facilitate implications for practicing therapists. To probe the moderation further, the Johnson-Neyman procedure (Bauer \& Curran, 2005) was applied to a linear model without random effects, as the estimates of the significance of the predictor's slopes were comparable to the model with random intercept. This procedure identified the points along the moderator (change in the global alliance) where the relationship between confrontations and alliance ruptures transitions from statistical non-significance to significance. A KruskalWallis test was used for the control analysis regarding the difference in fraction of sessions occupied by ruptures in dyads with a positive and negative change in the global alliance after therapy onset, since assumptions of heteroscedasticity for a t-test were violated. 
Post-hoc created Hypothesis 3 (Macro Level): Associations with outcome

Results of the first analysis regarding hypothesis 1 suggested that therapists make parallel use of resolution attempts and confrontations; this association varied a lot over the sample (see result section below for details). Therefore, we tried to probe the influence of confrontations (regarding discrepancy between patient and therapist) when used during a resolution attempt at any point during the two investigated sessions on the therapy outcome. As assumptions of heteroscedasticity for an ANOVA were violated, so Kruskal-Wallis tests were used to predict therapy outcome with a variable of three categories: no resolution attempt, resolution attempt without confrontation, resolution attempt with confrontation. To control for confounding variables regarding demographics, Kruskal-Wallis tests were used when examining age and Chi-square tests of independence were used when analyzing gender and diagnoses.

\section{Results}

\section{Incidence of Therapists' Confrontations co-occurs with Alliance Ruptures and}

\section{Resolution Attempts - Hypothesis 1}

Estimates based on a generalized linear mixed model (random intercept) indicated a significant association between incidence of ruptures and/or resolution attempts and incidence of confrontation within the same five-minute sequence (Table 1). Type of noticeable change in the therapeutic alliance influenced the reliability of predicting the incidence of confrontation in the same sequence: Compared to no rupture and/or resolution attempt $(O R=0.62 ; 95 \%$ CI $[0.52-0.73])$, a sole rupture $(O R=1.84 ; 95 \%$ CI $[1.44-2.34])$ as well as a sole resolution attempt $(O R=8.90 ; 95 \%$ CI [2.82-39.49]) statistically predicted a significantly higher probability of a confrontation within the same five-minute sequence. However, while a rupture-resolution episode also significantly predicted confrontations (OR $=1.78 ; 95 \%$ CI [1.13-2.83]), significance $(p=.013)$ might have been a side-product of the sample size ( $N=1892)$, especially when regarding the Cohen’s kappa of .46. Confidence 
intervals of estimates of the different categories of noticeable changes in the therapeutic alliance varied substantially. A confrontation was markedly more likely to occur during a sole resolution attempt than during a sole rupture or a rupture-resolution episode. However, confidence intervals of the estimated probability of the incidence of a confrontation in relation to a sole resolution attempt were considerably larger. Therefore, sequences containing a sole resolution attempt may have varied substantially in therapists' parallel use of confrontation. Furthermore, estimates based on the generalized linear mixed model implied a significant random effect based on the clustering by patients regarding the confidence interval of the random intercept (inclusion or exclusion of zero by confidence intervals can be used as an estimate of the significance of random effects; Finch, Bolin, \& Kelley, 2016).

[Table 1 near here]

\section{Alliance Ruptures Predicted with Therapists' Confrontation and Change in Global}

\section{Alliance - Hypothesis 2}

[Table 2 near here]

The analysis based on a linear mixed effects model implied a moderation of the association between confrontations (regarding discrepancies within patient or between patient and patient's environment) and alliance ruptures given means of the two investigated sessions per therapy. The bigger the fraction of a session occupied by confrontations, the higher the fraction of a session occupied by ruptures; this association was significantly influenced by the change in the global alliance before the investigated sessions. (Table 2, Fixed Effects). However, change in the global alliance on its own was not significantly associated with ruptures. This might have been influenced by the notable confidence interval of the estimated slope of the change in the global alliance. A closer look at the regions of significance (Johnson-Neyman procedure, Bauer \& Curran, 2005) of the slope of confrontation depending on the change in the global alliance revealed a difference between therapies characterized by 
a negative change and therapies characterized by a positive change after therapy onset: A positive association between the fraction occupied by ruptures and confrontations was only found in the latter. Additionally, confidence intervals of the random intercept (Table 2, Random Effects) revealed no measurable effect of the clustering by therapists on the mean amount of ruptures per dyad in this sample. This result should be interpreted considering possible doubts about the sample size that might not have allowed a reliable estimate of the therapist effect (Schiefele et al., 2017). To explore the mean fraction of a session occupied by ruptures depending on the quality of the global alliance, a Kruskal-Wallis test was applied to examine differences between the dyads with a negative change in the global alliance and the dyads with a positive change in the global alliance. The two groups showed no significant difference regarding the amount of ruptures $\left[\chi^{2}(1)=0.05, p=.819\right]$.

\section{Therapy Outcome in Relation to Therapists' Usage of Confrontation during}

\section{Resolution Attempt - Hypothesis 3}

The results of a Kruskal-Wallis test suggest that the use of confrontation during a resolution attempt is associated with a positive effect on therapy outcome (see Figure 2). There was a significant effect of resolution attempt/confrontation on therapy outcome at the $p<.05$ level for the three conditions $\left[\chi^{2}(2)=6.64, p=.036\right]$. Post hoc comparisons (Dunn-Bonferronitest) indicated that the mean score for the resolution attempt with confrontation condition ( $M$ $=0.85, S D=0.78$ ) was significantly different from that for the no resolution attempt condition $(M=0.33, S D=0.51)$. However, the resolution attempt without confrontation condition $(M=0.54, S D=0.65)$ did not significantly differ from the resolution attempt with confrontation and no resolution attempt conditions. Taken together, these results suggest that when therapists use confrontation (regarding discrepancy between patient and therapist) during a resolution attempt, therapy outcomes are better than no use of a resolution attempt at all or no use of a confrontation parallel to a resolution attempt. Further, Kruskal-Wallis tests 
revealed no additional differences between the three groups regarding mean fraction of session occupied by therapists' confrontations used outside of a resolution attempt $\left[\chi^{2}(2)=\right.$ $3.06, p=.216]$ or mean fraction of session occupied by ruptures $\left[\chi^{2}(2)=0.77, p=.681\right]$. A one-way between subjects ANOVA also showed no difference regarding change in global alliance after therapy onset $[\mathrm{F}(2,73)=0.28, \mathrm{p}=.600]$.

[Figure 2 near here]

Analyses of missing data and demographics showed no confounding effect on the tested variables except by patients' age. The 14 therapies that lacked data to calculate the therapy outcome did not differ from the remaining 63 therapies regarding mean fraction of session occupied by confrontations used outside of a resolution attempt $\left[\chi^{2}(1)=0.57, p=\right.$ $.452]$, mean fraction of session occupied by ruptures $\left[\chi^{2}(1)=0.62, p=.432\right]$ or change in global alliance after therapy onset $[\mathrm{F}(1,73)=0.001, \mathrm{p}=.979]$. Concerning demographics, there were no significant differences when grouping patients based on absence of data or based on resolution attempt/confrontation by therapists when considering patients' gender or diagnoses. When regarding patients' age, grouping based on use of resolution/confrontation by therapists revealed no differences, while grouping based on absence of data pointed to a significantly older sample in the group that lacked data regarding therapy outcome $\left[\chi^{2}(1)=\right.$ $3.86, \mathrm{p}=.049]$. A chi-square test of independence suggested that the conditions no resolution attempt, resolution attempt with confrontation and resolution attempt without confrontation did not differ in their amount of missing data regarding therapy outcome $\left[\chi^{2}(2, N=90)=\right.$ 3.66, $\mathrm{p}=.160]$.

\section{Discussion}

The purpose of this study was to test three hypotheses regarding the impact of confrontations by therapists on the therapeutic alliance and therapy outcome. The first hypothesis could be supported by our results on the micro level: All types of examined confrontations were related to ruptures within the same five-minute sequence. Additionally, therapists used 
confrontations in parallel to resolution attempts especially when the resolution attempts referred to a rupture in a separate five-minute sequence. This result was unaccounted for by predictions before the analysis. The second hypothesis could not be supported on the macro level: the linear mixed model revealed a significant association between confrontations (regarding discrepancies within patient or between patient and patient's environment) and ruptures when looking at a global alliance that had improved after therapy onset. This result was incompatible with the postulated significant association only when considering dyads with a therapeutic alliance of decreased quality since therapy onset.

This body of results might by interpreted by means of the TZPD (Leiman \& Stiles, 2001; Ribeiro et al., 2013): Rupture in the presence of a good therapeutic relationship can be understood as a non-collaborative response by the patient if the therapist's confrontation exceeds the patient's TZPD. To revise part of our introduction, a rupture after a therapist's confrontation may not have to be a marker of an unsuccessful confrontation as the possible exceeding of the patient's TZPD might be a reaction to be expected. The therapist's pushing beyond the patient's level might be a necessary process to be anticipated. Furthermore, therapists might have deliberately caused ruptures in the safe setting of a good therapeutic relationship to use them in a constructive and deliberate manner (which might include a validation of the patients' reaction to the confrontation and not a further pushing beyond their limits), as proposed by Coutinho et al. (2001) or Stiles, Caro Gabalda, and Ribeiro (2016). Alternatively, the association between confrontations and alliance ruptures can also be interpreted as therapists' hostile reactions in the presence of alliance ruptures. We cannot dismiss this alternative explanation because our methods did not test causality.

Rhodes, Hill, Thompson, and Elliott (1994) and Binder and Strupp (1997) provide further explanations when looking exclusively at the patient's side of the equation: patients communicate negative feelings directly and disagree with therapists if they feel safe and 
supported, while they are afraid to show their true colors when the therapeutic relationship is insufficient. Ruptures might not be as closely related to specific interventions in therapies with no security in the therapeutic relationship, since the patient's discontent cannot be expressed right when it is triggered but stays beneath the surface to reappear seemingly unrelated to the triggering therapist's intervention. On the other hand, if patients feel safe to immediately express their reactions, specific interventions time the incidence of ruptures. This might explain why dyads with a negative and a positive change in the global alliance since therapy onset did not fundamentally differ in their mean amount of alliance ruptures in our sample.

Nevertheless, a good therapeutic relationship appears not to be able to lessen the impact of a therapist's confrontation as far as the criteria used in this study are concerned in spite of therapist's expectations thereof (Moeseneder et al., 2017). Focusing on discrepancies within the patient or between patient and patient's environment seems to be risky (or to push beyond the patient's TZPD) even if the therapeutic relationship is secured before the intervention. By focusing on a discrepancy, therapists might restrict patients’ perceived freedom as the intervention implies restricted freedom regarding both sides of the focused discrepancy (as lies in the nature of a discrepancy). In the theory of psychological reactance (Brehm \& Brehm, 1981), this leads to reactance which might be operationalized as an alliance rupture as measured through the patient's behavior. Namely, a therapist's confrontation as in focusing on a discrepancy, might lead to anxiety in the patient because of a perceived restriction in freedom. This anxiety, in turn, may be expressed in an alliance rupture, which might even take on the look of a strongly hostile reaction by the patient. Thus, therapists might take up on this disclosure to work through the tension together with the patient, which might provoke new insight and reinforce the alliance (Safran, Muran, \& Eubanks-Carter, 2011; Stiles et al., 2016). 
The post-hoc hypothesis could be supported by the analysis on the macro level: Use of confrontation by therapists (regarding discrepancy between patient and therapist) during resolution attempts was associated with a better therapy outcome than using no confrontation during or no resolution attempts at all when examining whole therapies. This analysis was based on the results regarding the first hypothesis that showed a significant association between confrontations by therapists and their resolution attempts within the same fiveminute sequence, especially concerning resolution attempts referring to a rupture in a separate sequence. Maybe, if a rupture has already passed (in an earlier session or sequence), a confrontation at the beginning of the resolution attempt relating to the discrepancy between patient and therapist is necessary to structure the intervention and help the patient understand the topic of interest to the therapist. Furthermore, confrontations regarding a discrepancy between patient and therapist may be an integral part of successful resolution attempts, at least in our specific sample, as they seem to be associated with a significantly better therapy outcome. This possible enriching of resolutions attempts by confrontation seems to fit well into the concept of therapeutic metacommunication (Muran, 2017; Safran \& Muran, 2000) defined as “a communication about the communication process” (Muran, 2017, p. 4). A confrontation might help to regain perceived freedom over a strained interpersonal situation by verbalizing it if we revisit the above-mentioned theory of psychological reactance (Brehm \& Brehm, 1981) . This might in turn lead to a more constructive process, since the disclosure of previously silent strain on the relationship allows the patient to again be on equal footing with the therapist. Additionally, the therapist shows authenticity and competence by addressing a difficult topic frankly.

These different applications of the theory of psychological reactance might suggest qualitatively different kinds of confrontation, one of which could be defined by the personal involvement of the therapist within the focused discrepancy (when focusing on a discrepancy 
between patient and therapist), while the other excludes the therapist from the intervention (when focusing on discrepancies within patient or between patient and patient's environment). These two categories of confrontations might also be characterized by their respectively liberating and restricting nature. Furthermore, the former was relatively rarely rated in our sample considering its seemingly potent effect on the therapy outcome. Similarly, Hill et al. (2014) found that only 8\% of their rated immediacy events (defined as a mindful discussion of the therapeutic relationship by therapist and patient) related to ruptures. They suggested that it is easier to talk about feelings than ruptures and they noticed that therapists did not share their own feelings as often as they asked patients to talk about their feelings regarding the relationship. Various, similar aspects might explain the potential underuse of confrontation by therapists regarding a discrepancy between patient and therapist in our sample: Reluctance to talk about their own feelings as therapists, fear of further rupture and insufficient teaching of explicit utilization of alliance rupture in their training. Lastly, our analyses did not allow specifying safe or unsafe ways to confront when referring to a discrepancy between patient and therapist.

\section{Limitations}

One set of limitations arises as a consequence of the focus on two sessions per therapy that were in the middle third of therapy: implications regarding the beginning or end phase of therapy cannot be inferred from our study, as different processes regarding the interplay between confrontation and the therapeutic alliance might take place. Additionally, processes in the first third of therapy were not controlled for. It is therefore not possible to account for the influence of prior resolution attempts, which may have affected patients' expression of anxiety and hostility as well as therapists' deliberate work with alliance ruptures. Another set of limitations presents itself based on the measures. Firstly, the timing of confrontations and alliance ruptures could not be statistically checked because of the scales of measurements, so 
there is no certainty of confrontations causing ruptures or vice versa. Secondly, the Cohen’s kappa value regarding the 3RS-manual was arguably on the lower side, which suggests a cautious interpretation of the results on the micro level as they might have been a by-product of the sample size. Thirdly, based on the post-hoc nature of the third hypothesis, the outcome measure had to be constructed in an assembling fashion, because a substantial amount of data to construct the outcome variable was missing. This prevented the use of a singular measure for the score or a selection of measures based on a factor analysis and resulted in the use of all existing measures that were approximately constant over the time span of our therapies to maximize the statistical power. In regard to the sample size, it might not have allowed to reliably control for the therapist effect with the linear mixed effects model on the macro level. Lastly, no manipulation took place, as we focused on processes in a naturalistic setting, which in turn enhances the external validity of our study and might therefore facilitate implications for practicing therapists.

\section{Clinical Implications and Future Research}

From the practitioner's side, there are some implications: Firstly, confrontations might be risky (= trigger alliance ruptures) when they do not include the therapist as part of the focused discrepancy regardless of the prior quality of the therapeutic alliance. This means in turn, that alliance ruptures following a confrontation must not be interpreted as indicators for low therapeutic relationship quality. Alliance ruptures might surprisingly even be an embodiment of an open communication, in which patients feels safe enough to disagree or show discontent immediately after a relevant intervention. Secondly, the active and deliberate work with alliance ruptures might profit from an initial increase of the interpersonal tension (by making it explicit through a confrontation) so it can be cleanly and truly resolved. This explication might also make it easier to relate to again in the future, as it is assumed that alliance ruptures represent general dysfunctional interpersonal patterns of the patient (Safran 
\& Muran, 2006) and would thus probably reappear later in therapy. However, the shaping of such an intervention might be of great importance and has not been investigated in this study. Future research might therefore focus on systematic inquiry of strategies of shaping the focus on a discrepancy between therapist and patient. The concepts and methods relating to MOTR (Caspar, 2007) provide possibilities to operationalize the shaping of a confrontation and the direct relation of a confrontation to a patient's specific functioning. The use of MOTR and Plan Analysis might improve the prediction of the impact of confrontations on the therapeutic relationship and therapy outcome. Furthermore, future research might examine certain categories of resolution attempts such as immediate repair strategies and expressive resolution strategies and whether they differ in their inclusion of confrontation and association with therapy outcome. Additionally, confrontations might also be categorized (for example as proposed above based on the ex- or inclusion of the therapist in the focused discrepancy) and examined regarding different associations with the therapeutic alliance and outcome. Different operationalization of the quality of the therapeutic relationship, especially on a micro level, and its interplay with confrontation, ruptures and resolution attempts might suggest other patterns and implications. The study of whole therapies (instead of many therapies with only two sessions per dyad here) might shed light on such patterns on a more microscopic level.

\section{Conclusion}

Confrontations seem to have the potential of pushing patients beyond their limits, even in the environment of a good therapeutic alliance. Confrontations might also be involved in important processes of utilizing alliance ruptures. These conclusions need to be further tested, especially by applying different scales of measurement to examine assumptions about the direction of causality. Our results suggest that therapists might not be afraid to confront, if they listen willingly and react mindfully to discontent and disagreement in their patients. 


\section{References}

American Psychiatric Association. (2000). DSM-IV-TR: Diagnostic and statistical manual of mental disorders, text revision. Washington, DC: American Psychiatric Association.

Bates, D., Mächler, M., Bolker, B., \& Walker, S. (2014). Fitting linear mixed-effects models using lme4. arXiv preprint arXiv:1406.5823.

Bauer, D. J., \& Curran, P. J. (2005). Probing interactions in fixed and multilevel regression: Inferential and graphical techniques. Multivariate Behavioral Research, 40(3), 373400. doi:10.1207/s15327906mbr4003_5

Binder, J. L., \& Strupp, H. H. (1997). “Negative process”: A recurrently discovered and underestimated facet of therapeutic process and outcome in the individual psychotherapy of adults. Clinical Psychology: Science and Practice, 4(2), 121-139. doi:10.1111/j.1468-2850.1997.tb00105.x

Boardman, T., Catley, D., Grobe, J. E., Little, T. D., \& Ahluwalia, J. S. (2006). Using motivational interviewing with smokers: Do therapist behaviors relate to engagement and therapeutic alliance? Journal of Substance Abuse Treatment, 31(4), 329-339. doi:10.1016/j.jsat.2006.05.006

Bordin, E. S. (1979). The generalizability of the psychoanalytic concept of the working alliance. Psychotherapy: Theory, Research and Practice, 16(3), 252-260. doi:10.1037/h0085885

Brehm, S. S., \& Brehm, J. W. (1981). Psychological reactance: A theory of freedom and control. New York: Academic Press.

Cardillo, J. E., \& Smith, A. (1994). Psychometric issues. In Kiresuk, T. J., Smith, A., \& Cardillo, J. E. (Eds.), Goal attainment scaling: Applications, theory, and measurement (pp. 173-212). Mahwah NJ: Lawrence Erlbaum. 
Caspar, F. (2007). Plan Analysis. In Eells, T. D. (Ed.), Handbook of psychotherapy case formulations (2 ed., pp. 221-289). New York: Guilford Press.

Cicchetti, D. V. (1994). Guidelines, criteria, and rules of thumb for evaluating normed and standardized assessment instruments in psychology. Psychological Assessment, 6(4), 284-290. doi:10.1037/1040-3590.6.4.284

Claiborn, C. D., Goodyear, R. K., \& Horner, P. A. (2002). Feedback. In Norcross, J. C. (Ed.), Psychotherapy relationships that work: Therapist contributions and responsiveness to patients (pp. 217-233). Oxford: Oxford University Press.

Cohen, J. (1992). A power primer. Psychological Bulletin, 112(1), 155-159. doi:10.1037/0033-2909.112.1.155

Coutinho, J., Ribeiro, E., Hill, C., \& Safran, J. (2011). Therapists' and clients' experiences of alliance ruptures: A qualitative study. Psychotherapy Research, 21(5), 525-540. doi:10.1080/10503307.2011.587469

Engle, D., \& Arkowitz, H. (2008). Viewing resistance as ambivalence: Integrative strategies for working with resistant ambivalence. Journal of Humanistic Psychology, 48(3), 389-412. doi:10.1177/0022167807310917

Eubanks, C. F., Muran, J. C., \& Safran, J. D. (2015). Rupture resolution rating system (3RS): Manual. Unpublished manuscript, Mount Sinai-Beth Israel Medical Center, New York.

Figlioli, P. (2016). Wie nett soll Psychotherapie sein?: Der dynamische Prozess der Konfrontation und der Komplementarität in guten und schlechten Therapien [How nice should psychotherapy be?: The dynamic process of confrontation and complementarity in good and bad therapies]. (Unpublished doctoral dissertation), Universität Bern, Bern, Switzerland. 
Finch, W. H., Bolin, J. E., \& Kelley, K. (2016). Multilevel modeling using R. Boca Raton, FL: Crc Press.

First, M. B., Spitzer, R. L., Gibbon, M., \& Williams, J. B. (1995a). Structured clinical interview for DSM-IV axis I disorders. New York: New York State Psychiatric Institute.

First, M. B., Spitzer, R. L., Gibbon, M., Williams, J. B., Davies, M., Borus, J., . . . Rounsaville, B. (1995b). The structured clinical interview for DSM-III-R personality disorders (SCID-II). Part II: Multi-site test-retest reliability study. Journal of Personality Disorders, 9(2), 92-104.

Fitzmaurice, G. M., Laird, N. M., \& Ware, J. H. (2012). Applied longitudinal analysis. New Jersey: John Wiley \& Sons.

Flückiger, C., Caspar, F., Holtforth, M. G., \& Willutzki, U. (2009). Working with patients’ strengths: A microprocess approach. Psychotherapy Research, 19(2), 213-223. doi:10.1080/10503300902755300

Flückiger, C., Regli, D., Zwahlen, D., Hostettler, S., \& Caspar, F. (2010). The Bern Post Session Report 2000, patient and therapist versions: Measuring psychotherapeutic processes. Zeitschrift für Klinische Psychologie und Psychotherapie, 39(2), 71-79.

Gollwitzer, M., Christ, O., \& Lemmer, G. (2014). Individual differences make a difference: On the use and the psychometric properties of difference scores in social psychology. European Journal of Social Psychology, 44(7), 673-682.

Grawe, K. (2004). Psychological therapy. Toronto: Hogrefe Publishing.

Grawe, K., Caspar, F., \& Ambühl, H. (1990). Die Berner Therapievergleichsstudie: Zusammenfassung und Schlussfolgerungen [The Bernese comparative trial: Summary and conlusions]. Zeitschrift für Klinische Psychologie, 19(4), 362-376. 
Grosse Holtforth, M., \& Grawe, K. (2003). Der Inkongruenzfragebogen (INK) [Incongruence questionnaire (INK)]. Zeitschrift für Klinische Psychologie und Psychotherapie, 32(4), 315-323. doi:10.1026/0084-5345.32.4.315

Hill, C. E. (1978). Development of a counselor verbal response category. Journal of Counseling Psychology, 25(5), 461-468. doi:10.1037/0022-0167.25.5.461

Hill, C. E., Gelso, C. J., Chui, H., Spangler, P. T., Hummel, A., Huang, T., . . B Bhatia, A. (2014). To be or not to be immediate with clients: The use and perceived effects of immediacy in psychodynamic/interpersonal psychotherapy. Psychotherapy Research, 24(3), 299-315. doi:10.1080/10503307.2013.812262

Hill, C. E., Nutt-Williams, E., Heaton, K. J., Thompson, B. J., \& Rhodes, R. H. (1996). Therapist retrospective recall impasses in long-term psychotherapy: A qualitative analysis. Journal of Counseling Psychology, 43(2), 207-217. doi:10.1037/00220167.43.2.207

Horvath, A. O. (2000). The therapeutic relationship: From transference to alliance. Journal of Clinical Psychology, 56(2), 163-173. doi:10.1002/(SICI)10974679(200002)56:2\%3C163::AID-JCLP3\%3E3.0.CO;2-D

Itten, S., \& Grawe, K. (2003). VLB-Veränderungsfragebogen für Lebensbereiche [Change Questionnaire in Life Domains]. In Brähler, E., Schumacher, J., \& Strauß, B. (Eds.), Diagnostische Verfahren in der Psychotherapie (pp. 382-384). Göttingen: Hogrefe.

Jerusalem, M., \& Schwarzer, R. (1999). Skala zur allgemeinen Selbstwirksamkeitserwartung [Generalized Self-Efficacy Scale]. Berlin: Freie Universität Berlin.

Klaghofer, R., \& Brähler, E. (2001). Konstruktion und Teststatistische Prüfung einer Kurzform der SCL-90-R [Construction and test statistical evaluation of a short version of the SCL-90-R]. Zeitschrift für Klinische Psychologie, Psychiatrie und Psychotherapie, 49(2), 115-124. 
Kramer, U., Kolly, S., Berthoud, L., Keller, S., Preisig, M., Caspar, F., . . Despland, J.-N. (2014). Effects of motive-oriented therapeutic relationship in a ten-session general psychiatric treatment of borderline personality disorder: A randomized controlled trial. Psychotherapy and Psychosomatics, 83(3), 176-186. doi:10.1159/000358528

Lambert, M. J., \& Barley, D. E. (2001). Research summary on the therapeutic relationship and psychotherapy outcome. Psychotherapy: Theory, Research, Practice, Training, 38(4), 357-361. doi:10.1037/0033-3204.38.4.357

Leiman, M., \& Stiles, W. B. (2001). Dialogical sequence analysis and the zone of proximal development as conceptual enhancements to the assimilation model: The case of Jan revisited. Psychotherapy Research, 11(3), 311-330. doi:10.1080/713663986

Meystre, C., Pascual-Leone, A., de Roten, Y., Despland, J.-N., \& Kramer, U. (2015). What interventions facilitate client progress through the assimilation model? A task analysis of interventions in the psychodynamic treatment of depression. Psychotherapy Research, 25(4), 484-502. doi:10.1080/10503307.2014.921352

Moeseneder, L., Figlioli, P., \& Caspar, F. (2017). Confronting patients: Therapists’ model of a responsiveness based approach. Journal of Contemporary Psychotherapy, 48(2), 6167. doi:10.1007/s10879-017-9371-X

Moyers, T. B., Miller, W. R., \& Hendrickson, S. M. L. (2005). How does motivational interviewing work? Therapist interpersonal skill predicts client involvement within motivational interviewing sessions. Journal of Consulting and Clinical psychology, 73(4), 590-598. doi:10.1037/0022-006X.73.4.590

Muran, J. C. (2017). Confessions of a New York rupture researcher: An insider's guide and critique. Psychotherapy Research, 1-14. doi:10.1080/10503307.2017.1413261

Norcross, J. C. (2002). Psychotherapy relationships that work: Therapist contributions and responsiveness to patients. New York: Oxford University Press. 
Polcin, D. L. (2006). Reexamining confrontation and motivational interviewing. Addictive Disorders and their Treatment, 5(4), 201-209. doi:10.1097/01.adt.0000205048.44129.6a

R Development Core Team. (2014). R: A language and environment for statistical computing. Vienna, Austria: R Foundation for Statistical Computing.

Rhodes, R. H., Hill, C. E., Thompson, B. J., \& Elliott, R. (1994). Client retrospective recall of resolved and unresolved misunderstanding events. Journal of Counseling Psychology, 41(4), 473-483. doi:10.1037/0022-0167.41.4.473

Ribeiro, E., Ribeiro, A. P., Gonçalves, M. M., Horvath, A. O., \& Stiles, W. B. (2013). How collaboration in therapy becomes therapeutic: The therapeutic collaboration coding system. Psychology and Psychotherapy: Theory, Research and Practice, 86(3), 294314. doi:10.1111/j.2044-8341.2012.02066.x

Sachse, R. (2003). Klärungsorientierte Psychotherapie [Clarification-oriented psychotherapy]. Göttingen: Hogrefe

Safran, J. D., Crocker, P., McMain, S., \& Murray, P. (1990). Therapeutic alliance rupture as a therapy event for empirical investigation. Psychotherapy: Theory, Research, Practice, Training, 27(2), 154-165. doi:10.1037/0033-3204.27.2.154

Safran, J. D., \& Muran, J. C. (2000). Negotiating the therapeutic alliance: A relational treatment guide. New York: Guilford Press.

Safran, J. D., \& Muran, J. C. (2006). Has the concept of the therapeutic alliance outlived its usefulness? Psychotherapy: Theory, Research, Practice, Training, 43(3), 286-291. doi:10.1037/0033-3204.43.3.286

Safran, J. D., Muran, J. C., \& Eubanks-Carter, C. (2011). Repairing alliance ruptures. Psychotherapy, 48(1), 80-87. doi:10.1037/a0022140 
Safran, J. D., Muran, J. C., Samstag, L. W., \& Stevens, C. (2001). Repairing alliance ruptures. Psychotherapy: Theory, Research, Practice, Training, 38(4), 406-412. doi:10.1037/0033-3204.38.4.406

Stiles, W. B., Caro Gabalda, I., \& Ribeiro, E. (2016). Exceeding the therapeutic zone of proximal development as a clinical error. Psychotherapy, 53(3), 268-272. doi:10.1037/pst0000061

Strong, T., \& Zeman, D. (2010). Dialogic considerations of confrontation as a counseling activity: An examination of Allen Ivey's use of confronting as a microskill. Journal of Counseling and Development, 88(3), 332-339. doi:10.1002/j.15566678.2010.tb00030.x

Williams, D. C., \& Levitt, H. M. (2007). A qualitative investigation of eminent therapists' values within psychotherapy: Developing integrative principles for moment-tomoment psychotherapy practice. Journal of Psychotherapy Integration, 17(2), 159184. doi:10.1037/1053-0479.17.2.159 
Table 1

Generalized Linear Mixed Model

\begin{tabular}{lccccr}
\hline Fixed Effects $^{a}$ & Estimate & SE & CI & Z & $(>|\mathrm{z}|)$ \\
\hline Intercept (no Rupture/ & 0.48 & 0.09 & $-0.65,-0.31$ & -5.63 & $<.001$ \\
Resolution Attempt) & & & & & \\
Rupture Only & 0.60 & 0.12 & $0.36,0.85$ & 4.88 & $<.001$ \\
Resolution Attempt Only & 2.19 & 0.65 & $1.03,3.68$ & 3.35 & $<.001$ \\
Rupture-Resolution & 0.58 & 0.24 & $0.12,1.04$ & 2.47 & .013
\end{tabular}

Episode $^{\mathrm{b}}$

Random Effect ${ }^{c} \quad$ Std.De

$0.55 \quad 0.41,0.72$

${ }^{\mathrm{a}}$ Marginal R-squared $=0.03$. $^{\mathrm{b}}$ Rupture and associated resolution attempt within same five-minute sequence.

${ }^{\mathrm{c}}$ Conditional R-squared $=0.11$.

Table 2

Linear Mixed Model

\begin{tabular}{lccccr}
\hline Fixed Effects $^{\mathrm{a}}$ & Estimate & $\mathrm{SE}$ & $\mathrm{CI}$ & t value & $\operatorname{Pr}(>|\mathrm{t}|)$ \\
\hline Intercept & 26.66 & 2.05 & $22.55,31.06$ & 12.99 & $<.001$ \\
Confrontation & 1.66 & 5.22 & $0.16,3.19$ & 1.18 & .038 \\
Change in GA & 6.14 & 0.78 & $-3.99,16.55$ & 2.12 & .245 \\
Confrontation $*$ & 3.61 & 1.25 & $1.45,5.96$ & 2.89 & .006 \\
Change in GA & & & & & \\
Random Effects $^{\mathrm{b}}$ & Std.Dev & & CI & \\
\hline Intercept & 4.37 & & $0.00,10.67$ & \\
${ }^{\mathrm{a}}$ Marginal R-squared $=0.20 .{ }^{\mathrm{b}}$ Conditional R-squared $=0.42$. & &
\end{tabular}




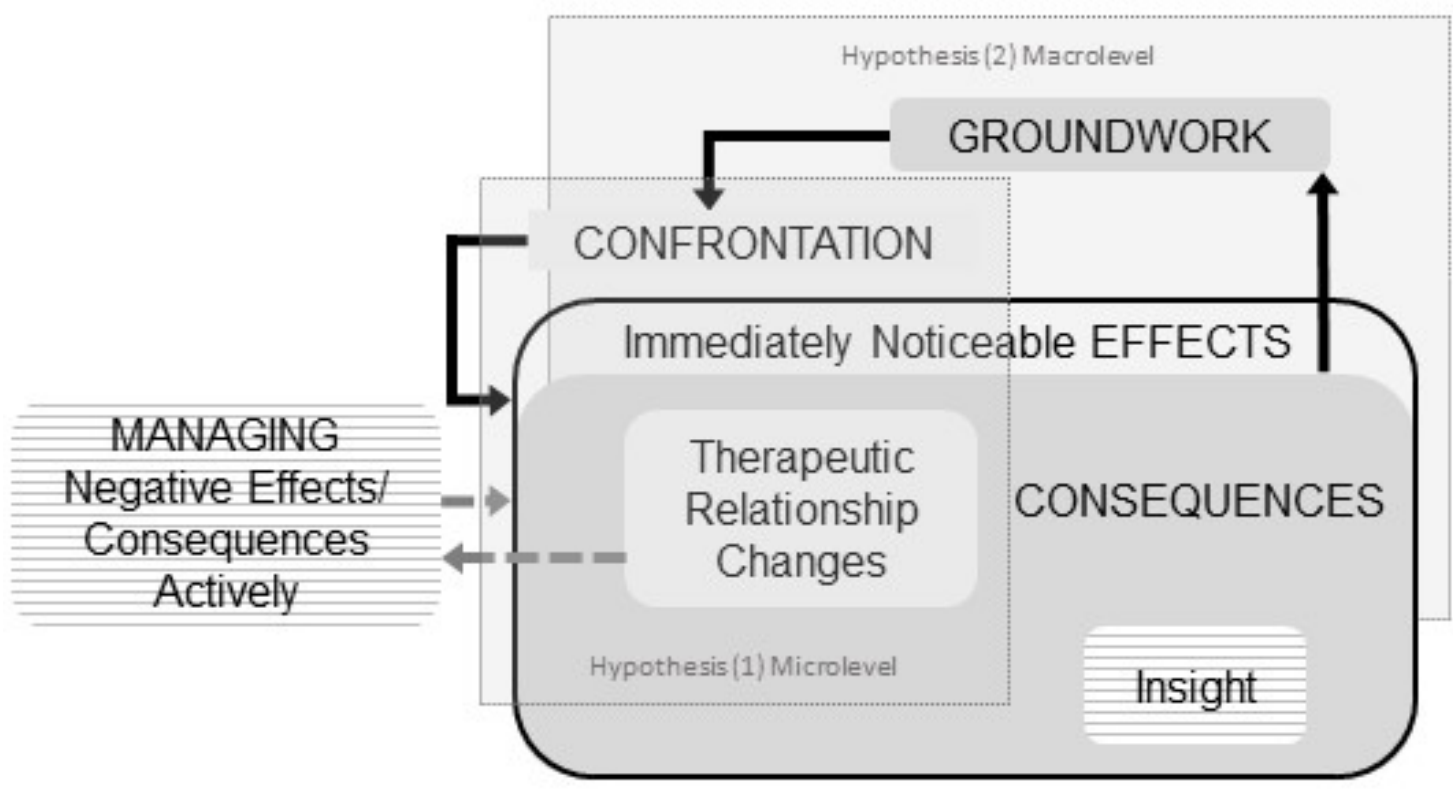

Figure 1. A tentative conceptual model of a Responsiveness based approach when confronting patients as postulated by therapists in interviews (modified with permission from Moeseneder, Figlioli, \& Caspar, 2017). Arrows illustrate preceding themes/subcategories affecting subsequent themes/subcategories. Horizontally shaded boxes include partial occasional processes. Boxes in dotted lines relate to a priori generated hypotheses in present study.

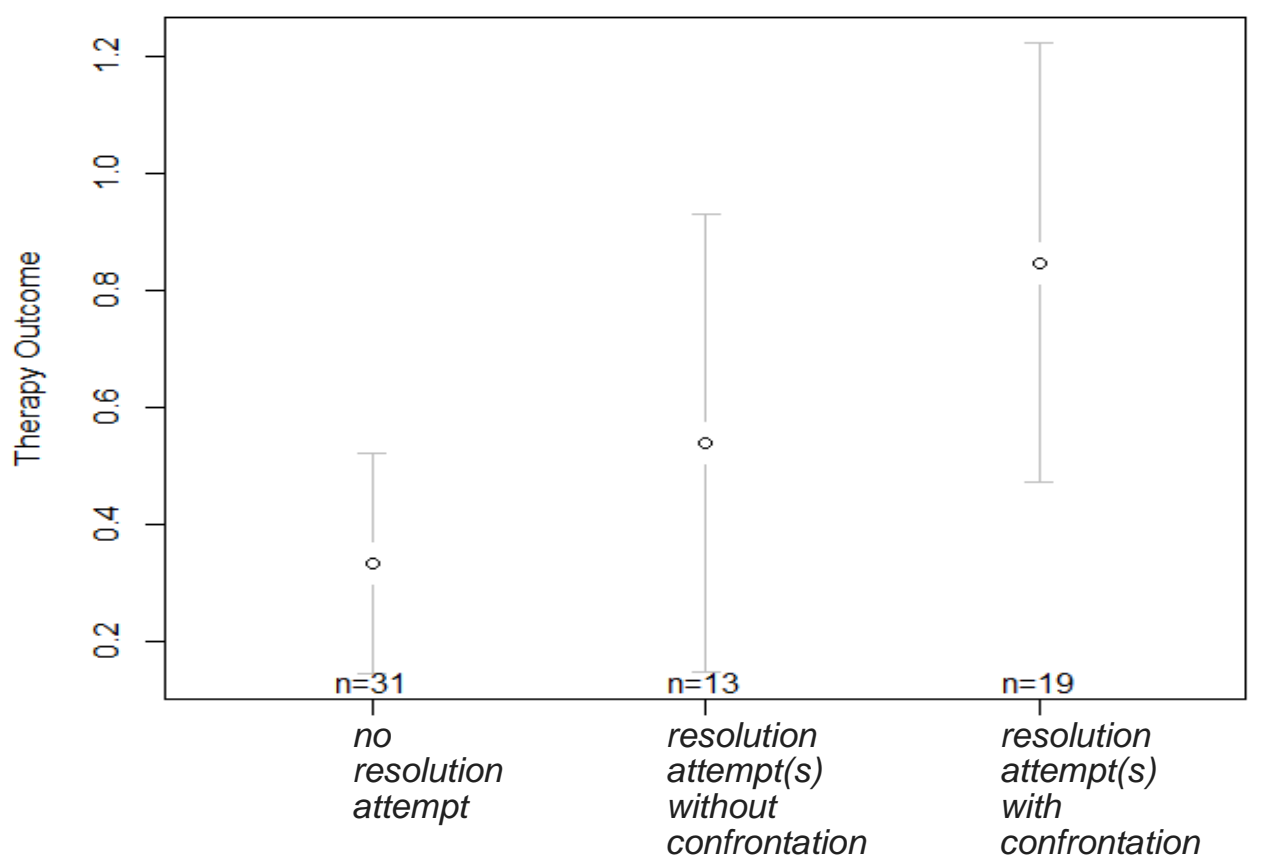

Figure 2. Therapy outcome (mean difference of measurements during the two investigated sessions and posttherapy divided by the pooled deviation of both times of measurements) with error bars by resolution attempt/confrontation. 